Article

\title{
On Dynamic Systems in the Frame of Singular Function Dependent Kernel Fractional Derivatives
}

\author{
Thabet Abdeljawad $1,2, * \mathbb{C}$, Fadila Madjidi ${ }^{3}$, Fahd Jarad ${ }^{4, *}$ and Ndolane Sene ${ }^{5}$ \\ 1 Department of Mathematics and General Sciences, Prince Sultan University, P. O. Box 66833, \\ Riyadh 11586, Saudi Arabia \\ 2 Department of Medical Research, China Medical University, Taichung 40402, Taiwan \\ 3 Department of Mathematics, University of Mohamed Boudiaf-PB 166, M'sila 28000, Algeria; \\ fadila.madjidi@univ-msila.dz \\ 4 Department of Mathematics, Çankaya University, 06790 Etimesgut, Ankara, Turkey \\ 5 Laboratoire Lmdan, Dèpartement de Mathèmatiques de la Dècision, Universitè Cheikh Anta Diop de Dakar, \\ Facultè des Sciences Economiques et Gestion, Dakar Fann BP 5683, Senegal; ndolanesene@yahoo.fr \\ * Correspondence: tabdeljawad@psu.edu.sa (T.A.); fahd@cankaya.edu.tr (F.J.)
}

Received: 15 August 2019; Accepted: 9 October 2019; Published: 11 October 2019

\begin{abstract}
In this article, we discuss the existence and uniqueness theorem for differential equations in the frame of Caputo fractional derivatives with a singular function dependent kernel. We discuss the Mittag-Leffler bounds of these solutions. Using successive approximation, we find a formula for the solution of a special case. Then, using a modified Laplace transform and the Lyapunov direct method, we prove the Mittag-Leffler stability of the considered system.
\end{abstract}

Keywords: generalized fractional operators; Mittag-Leffler bound; Mittag-Leffler stability

\section{Introduction and Preliminaries}

Fractional calculus, being considered as the generalization of the calculus dealing with integration and differentiation of functions with integer order, has fetched the interest of many researchers working on different scopes of science and engineering [1-5].

Due to the spatial non-locality of the operators with fractional orders, these operators have become significant tools that enable researchers to bring new aspects to the dynamics of non-local complex systems [1-8]. In addition, there has been an intensive interest in dealing with differential equations embodying derivatives with fractional order from many point of views including the qualitative, theoretical and numerical aspects [1-3] and studying the existence and uniqueness of solutions of differential equations in the frame of the traditional fractional derivative has been tackled in many works (see [9] and the references therein).

The generalization of the traditional fractional operators has been an important issue and it has been tackled by many researchers. The author in [10,11] proposed what was called the generalized fractional operators. These operators involve two parameters and are reduced to well known operators such as the Riemann-Liouville and the Hadamard fractional operators [3,12] when one of these parameters approaches some values. In addition, the fractional derivatives considered there were also modified in the Caputo settings so that they merge the Caputo fractional derivative and the Caputo-Hadamard one [3,13-16]. Nevertheless, the fractional operators considered [10,11] are still special cases of the so called the fractional operators of a function with respect to another function or fractional operators with function dependent kernels [2,3]. The Caputo modification of the fractional derivatives of functions with respect to another function was presented in [17]. In that work, the authors considered a modified Laplace transform that enables the scientists to deal with such operators. 
In addition, in the literature one can find more generalized types of fractional derivatives, among which the so called tempered fractional derivatives attracted many researchers. It is worth mentioning that the tempered fractional derivatives utilize an exponential tempering to the power-law kernel. For more information on these derivatives we refer to [18] and the references cited there.

In spite of the fact that there are many types of traditional fractional operators, these operators, having singularities in their kernels, might not be the most ideal operator a researcher could choose for the sake of better understanding and modeling the real world phenomena under investigation. For this reason, some researchers considered and explored new types of fractional operator embodying non-singular kernels that still possess the non-locality property, which is the most fundamental property of the fractional operators [19-24].

The main feature of the fractional derivatives is that the power law kernel codes the flexibility of an infinite number of relaxation times. This is one of the main reasons that fractional differential equations provide good experimental fits to a broad range of time scales [25]. The singularities from the power-law kernels can be computationally dealt with using Lubich's corrections and non-uniform grids.

There has been a large number of articles from those that discussed the extension of qualitative properties of differential equations with integer order derivatives, to those including fractional derivatives. Among these qualitative properties, stability plays a significant role [26-36]. In this article, we study some qualitative properties of dynamic systems in the frame of fractional derivatives involving a function dependent kernel. We discuss the existence and uniqueness of a nonlinear Cauchy problem and obtain the solution in the linear case. Then, we investigate the Mittag-Leffler stability of these systems.

Before we start presenting the main results, we state some definitions, lemmas and properties from the literature.

The left Riemann-Liouville fractional integral of order $\varepsilon$, where $\varepsilon \in \mathbb{C}, \operatorname{Re}(\varepsilon)>0$ has the following form

$$
\left({ }_{a} I^{\varepsilon} f\right)(t)=\frac{1}{\Gamma(\varepsilon)} \int_{a}^{t}(t-v)^{\varepsilon-1} f(v) d v .
$$

The left Riemann-Liouville fractional derivative of order $\varepsilon$, where, $\varepsilon \in \mathbb{C}, \operatorname{Re}(\varepsilon) \geq 0$ reads

$$
\left({ }_{a} D^{\varepsilon} f\right)(t)=\left(\frac{d}{d t}\right)^{n}\left({ }_{a} I^{n-\varepsilon} f\right)(t), \quad n=[\varepsilon]+1 .
$$

The left Caputo fractional derivative of order $\varepsilon, \operatorname{Re}(\varepsilon) \geq 0$ takes the form

$$
\left({ }_{a} D^{\varepsilon} f\right)(t)=\left({ }_{a} I^{n-\varepsilon} f^{(n)}\right)(t), \quad n=[\varepsilon]+1 .
$$

The left Hadamard fractional integral of order $\varepsilon \in \mathbb{C}, \operatorname{Re}(\varepsilon)>0$ is defined by

$$
\left({ }_{a} \mathcal{J}^{\varepsilon} f\right)(t)=\frac{1}{\Gamma(\varepsilon)} \int_{a}^{t}(\ln t-\ln v)^{\varepsilon-1} \frac{f(v)}{v} d v
$$

while the left Hadamard fractional derivative of order $\varepsilon \in \mathbb{C}, \operatorname{Re}(\varepsilon) \geq 0$ is given as

$$
\left({ }_{a} \mathcal{D}^{\varepsilon} f\right)(t)=\left(t \frac{d}{d t}\right)^{n}\left({ }_{a} \mathcal{J}^{n-\varepsilon} f\right)(t), \quad n=[\varepsilon]+1 .
$$

In $[13,14,16]$, the left Caputo-Hadamard fractional derivatives of order $\varepsilon \in \mathbb{C}, \operatorname{Re}(\varepsilon) \geq 0$ were defined as

$$
\left({ }_{a}^{C} \mathcal{D}^{\varepsilon} f\right)(t)={ }_{a} \mathcal{D}^{\varepsilon}\left[f(u)-\sum_{i=0}^{n-1} \frac{\delta^{i} f(a)}{i !}\left(\ln \frac{v}{a}\right)^{i}\right](t), \quad \delta=t \frac{d}{d t}
$$


and in the space $A C_{\delta}^{n}[a, b]=\left\{f:[a, b] \rightarrow \mathbb{C}: \delta^{n-1}[f(x)] \in A C[a, b]\right\}$ equivalently by

$$
\left({ }_{a}^{C} \mathcal{D}^{\varepsilon} f\right)(t)=\left({ }_{a} \mathcal{J}^{n-\varepsilon}\left(v \frac{d}{d v}\right)^{n} f\right)(t), \quad n=[\varepsilon]+1,
$$

and for $a<b, c \in \mathbb{R}$ and $1 \leq p<\infty$, define the function space

$$
X_{c}^{p}(a, b)=\left\{f:[a, b] \rightarrow \mathbb{R}:\|f\|_{X_{c}^{p}}=\left(\int_{a}^{b}\left|t^{c} f(t)\right|^{p} \frac{d t}{t}\right)^{1 / p}<\infty\right\} .
$$

For $p=\infty,\|f\|_{X_{c}^{p}}=\operatorname{ess~sup~}_{a \leq t \leq b}\left[t^{c}|f(t)|\right]$. The generalized left fractional integrals in the sense of how they are defined in [10] have the form

$$
\left({ }_{a} I^{\varepsilon, \rho} f\right)(t)=\frac{1}{\Gamma(\varepsilon)} \int_{a}^{t}\left(\frac{t^{\rho}-v^{\rho}}{\rho}\right)^{\varepsilon-1} \frac{f(v) d v}{v^{1-\rho}} .
$$

Remark 1. If $\rho=1$ the integrals in (8) become the Riemann-Liouville fractional integrals (1) and when $\rho \rightarrow 0$ (8) become the Hadamard fractional integrals (4).

The left generalized fractional derivatives of order $\varepsilon>0$ are defined in [11] as

$$
\left({ }_{a} D^{\varepsilon, \rho} f\right)(t)=\gamma^{n}\left({ }_{a} I^{n-\varepsilon, \rho} f\right)(t)=\frac{\gamma^{n}}{\Gamma(n-\varepsilon)} \int_{a}^{t}\left(\frac{t^{\rho}-v^{\rho}}{\rho}\right)^{n-\varepsilon-1} \frac{f(v) d u}{v^{1-\rho}}
$$

where $\rho>0$ and where $\gamma=t^{1-\rho} \frac{d}{d t}$.

Remark 2. Once $\rho=1$ the derivatives in (9) reduce to the Riemann-Liouville fractional derivatives (2). Whereas, taking the limit of (9) as $\rho \rightarrow 0$ one obtains the Hadamard fractional derivatives (5).

The Caputo modifications of the left generalized fractional derivatives were proposed in [15] to have the form

$$
\left({ }_{a}^{C} D^{\varepsilon, \rho} f\right)(t)=\left({ }_{a} I^{n-\varepsilon, \rho} \gamma^{n} f\right)(t)=\frac{1}{\Gamma(n-\varepsilon)} \int_{a}^{t}\left(\frac{t^{\rho}-v^{\rho}}{\rho}\right)^{n-\varepsilon-1} \gamma^{n} \frac{f(v) d v}{v^{1-\rho}} .
$$

Remark 3. Replacing $\rho$ by 1 in (10) the Caputo fractional derivatives (3) are obtained and approaching $\rho$ to 0 in (10) gives the Caputo-Hadamard fractional derivatives (6).

Definition 1 ([3]). The $k$-left-fractional integral operator is defined by

$$
{ }_{a} I^{\varepsilon, k} f(x)=\frac{1}{\Gamma(\varepsilon)} \int_{a}^{x}(k(x)-k(t))^{\varepsilon-1} f(t) k^{\prime}(t) d t,
$$

where $\varepsilon \in \mathbb{C}, \operatorname{Re}(\varepsilon) \geq 0$.

Remark 4. We should remark that if $k(x)=x$, the left Riemann-Liouville fractional integrals (1) are obtained. If $k(x)=\ln x$, the Hadamard integrals (4) are obtained and at last when $k(x)=\frac{x^{\rho}}{\rho}$ the generalized fractional integrals in the sense of Katugampola (8) are obtained. 
Definition 2 ([17]). The $k$-left-fractional derivative of order $\varepsilon \in \mathbb{C}, \operatorname{Re}(\varepsilon) \geq 0$ in the Caputo setting is defined by

$$
\begin{aligned}
{ }_{a}^{C} D^{\varepsilon, k} f(x) & =\left(a I^{n-\varepsilon, k}\right)\left(T_{k}^{n} f\right)(x) \\
& =\frac{1}{\Gamma(n-\varepsilon)} \int_{a}^{x}(k(x)-k(t))^{n-\varepsilon-1} T_{k}^{n} f(t) k^{\prime}(t) d t
\end{aligned}
$$

where $n=[\operatorname{Re}(\varepsilon)]+1$, and $T_{g}^{n} f(x)=\left(\frac{D}{k^{\prime}(x)}\right)^{n} f(x)$ is the composition $n$ times of the differential operator $\frac{D}{k^{\prime}(x)}$.

Remark 5. One should notice that if one takes $k(x)=x$, the left Caputo fractional derivatives (3) are obtained. If $k(x)=\ln x$, the Caputo-Hadamard integrals (6) are obtained and finally if $k(x)=\frac{x^{\rho}}{\rho}$ the generalized Caputo fractional in the sense of Katugampola (10) are obtained. In particular, if $0<\varepsilon<1$, then

$$
\begin{aligned}
{ }_{a}^{C} D^{\varepsilon, k} f(x) & =\left({ }_{a} I^{1-\varepsilon, k}\right) T_{k} f(x) \\
& =\frac{1}{\Gamma(1-\varepsilon)} \int_{a}^{x}(k(x)-k(t))^{-\varepsilon} f^{\prime}(t) d t .
\end{aligned}
$$

In the Riemann-Liouville setting we have [3]

$$
\begin{aligned}
{ }_{a} D^{\varepsilon, k} f(x) & =T_{k}\left({ }_{a} I^{1-\varepsilon, k}\right) f(x) \\
& =\frac{T_{k}}{\Gamma(1-\varepsilon)} \int_{a}^{x}(k(x)-k(t))^{-\varepsilon} f(t) k^{\prime}(t) d t .
\end{aligned}
$$

Lemma 1 ([3]). Let $\operatorname{Re}(\beta)>0, \operatorname{Re}(\varepsilon)>0$ and $k(t)$ be an increasing and positive monotone function on $(a, b]$ which has a continuous derivative $k^{\prime}(t)$ on $(a, b)$. Then,

$$
\left({ }_{a} I^{\varepsilon, k}(k(t)-k(a))^{\beta-1}\right)(x)=\frac{\Gamma(\beta)}{\Gamma(\beta+\varepsilon)}(k(x)-k(a))^{\beta+\varepsilon-1} .
$$

Lemma 2 ([3]). For a continuous real-valued function $f$ and an increasing and positive monotone function $k$ on $(a, b]$ with continuous derivative $k^{\prime}$ on $(a, b)$, we have

$$
{ }_{a} I^{\varepsilon, k}{ }_{a} I^{\beta, k} f(x)={ }_{a} I^{\varepsilon+\beta, k} f(x), \quad \varepsilon>0, \beta>0 .
$$

Lemma 3 ([17]). For $\varepsilon>0$ and $f$ and $k$ as desired we have

$$
{ }_{a} I^{\varepsilon, k} \underset{a}{C} D^{\varepsilon, k} f(x)=f(x)-\sum_{i=0}^{n-1} \frac{(k(x)-k(a))^{i}}{i !} T_{k}^{i} f(a) .
$$

In particular, if $0<\varepsilon<1$ then

$$
{ }_{a} I^{\varepsilon, k}{ }_{a}^{C} D^{\varepsilon, k} f(x)=f(x)-f(a) .
$$

This article is organized as follows: The second section discusses the existence uniqueness of solutions to a certain type of differential equations involving a fractional derivative with dependence on a kernel function and taking the advantage of successive approximation, we find the solution of a special case of the system under consideration. In third section, an upper bound of the solutions is obtained and the Mittag-Leffler stability of the considered system is debated. In the last section, conclusions and discussions are presented. 


\section{Existence and Uniqueness Theorems}

Consider the following fractional nonlinear Cauchy problem

$$
{ }_{a}^{C} D^{\varepsilon, k} x(t)=f(t, x(t)), x(a)=x_{a}, t \in(a, b],
$$

where $\varepsilon \in(0,1), f:[a, b) \times G, G$ an open subset of $\mathbb{R}$ or more generally of $\mathbb{C}$, and

$$
\begin{array}{r}
h(t)=f(t, x(t)) \in C_{\gamma, k}[a, b]=\left\{y:(a, b] \rightarrow \mathbb{R}:(k(t)-k(a))^{\gamma} y(t) \in C[a, b]\right\}, \\
0 \leq \gamma<1 .
\end{array}
$$

The space $C_{\gamma, k}[a, b]$ is a Banach space when it is endowed by the norm

$$
\|y\|=\sup _{t}\left|e^{-N(k(t)-k(a))}(k(t)-k(a))^{\gamma} y(t)\right|, \quad N>0,
$$

which is equivalent to the norm $\|y\|_{\gamma, k}=\sup _{t}\left|(k(t)-k(a))^{\gamma} y(t)\right|$. When $\gamma=0$ we accept that $C_{\gamma, \rho}[a, b]=C[a, b]$ the space of continuous functions on $[a, b]$ and when $k(t)=t$ we accept $C_{\gamma, k}[a, b]=$ $C_{\gamma}[a, b]$ (see [3], p. 4).

Definition 3. A function $x(t)$ is said to be a solution of the initial value problem (18) if

1. $(t, x(t)) \in D, D=[a, b] \times B, B=\{x \in \mathbb{R}:|x| \leq L\} \subset G, L>0$

2. $x(t)$ satisfies (18).

Theorem 1. The fractional initial value problem (18) has a unique solution in the space

$$
C_{\gamma, k}^{\varepsilon, 0}[a, b]=\left\{y(t) \in C[a, b]:{ }_{a}^{C} D^{\varepsilon, k} y(t) \in C_{\gamma, k}[a, b]\right\},
$$

with $0 \leq \gamma<1$ and $\gamma \leq \varepsilon$, provided that

$$
\frac{A}{N^{\varepsilon}}<1
$$

and $f$ satisfies the Lipschitzian condition

$$
\left|f\left(t, y_{1}\right)-f\left(t, y_{2}\right)\right| \leq A\left|y_{1}-y_{2}\right|, \quad A>0 .
$$

Proof. First let us prove the existence of a unique solution $y(t)$ in the space $C[a, b]$. Define the operator $\Psi: C[a, b] \rightarrow C[a, b]$ by

$$
(\Psi x)(t)=x_{a}+{ }_{a} I^{\varepsilon, k} f(t, x(t)) .
$$

where the space $C[a, b]$ is endowed with the norm $\|y\|_{C}=\sup _{t}\left|e^{-N(k(t)-k(a))} y(t)\right|$, which is equivalent to the sup norm. For any $y_{1}, y_{2} \in B$, by the help of the Lipschitz condition (21) and by writing $k(t)-k(a)=k(t)-k(s)+k(s)-k(a)$ we have

$$
\begin{gathered}
\left|e^{-N(k(t)-k(a))}\left(\Psi y_{1}(t)-\Psi y_{2}(t)\right)\right| \leq \\
\frac{A}{\Gamma(\varepsilon)} \int_{a}^{t} e^{-N \cdot K(t, s)} K(t, s)^{\varepsilon-1} k^{\prime}(s) d s\left\|y_{1}-y_{2}\right\|_{C} .
\end{gathered}
$$

Using the change of variable $u=K(t, s)=(k(t)-k(s))$, it follows that

$$
\left\|\Psi y_{1}-\Psi y_{2}\right\| \leq \frac{A}{\Gamma(\varepsilon)} \int_{0}^{(k(t)-k(a))} u^{\varepsilon-1} e^{-N u} d u\left\|y_{1}-y_{2}\right\|_{C},
$$


and hence, by using the definition of Gamma function we have,

$$
\left\|\Psi y_{1}-\Psi y_{2}\right\|_{C} \leq \frac{A}{N^{\varepsilon}}\left\|y_{1}-y_{2}\right\|_{C} .
$$

By assumption (20), the mapping $\Psi$ is a contraction and hence by Banach fixed point theorem it has a unique fixed point $x \in C\left[t_{0}, b\right]$. Moreover,

$$
\lim _{m \rightarrow \infty}\left\|T^{m} x_{a}-x\right\|_{C}=0 .
$$

From the definition of $\Psi, x$ has the form

$$
x(t)=x_{a}+{ }_{a} I^{\varepsilon, k} f(t, x(t)) .
$$

From Lemma 3 with $n=1$, it is clear that if $x$ satisfies the initial value problem (18), then it has the representation (27). Conversely, if $x$ has the representation (27), then clearly $x\left(t_{0}\right)=x_{0}$. By the help of the fact that for a continuous $z(t)$, we have

$$
{ }_{a}^{C} D^{\varepsilon, k} z(x)={ }_{a} D^{\varepsilon, k}[z(t)-z(a)](x), \quad{ }_{a} D^{\varepsilon, k}{ }_{a} I^{\varepsilon, k} z(t)=z(t),
$$

$x$ will satisfy the equation (18). Hence, $x$ has the representation (27) if and only if it satisfies the initial value problem (18). Finally, if $\|$.$\| denotes the norm defined in (19) then we have$

$$
\begin{aligned}
\left\|{ }_{a}^{C} D^{\varepsilon, k} T^{m} x_{a}-{ }_{a}^{C} D^{\varepsilon, k} x\right\| & \leq A\left\|T^{m} x_{a}-x\right\| \\
& \leq A\left(b^{\rho}-a^{\rho}\right)^{\gamma}\left\|T^{m} x_{a}-x\right\|_{C} .
\end{aligned}
$$

From (26), we conclude that $\lim _{m \rightarrow \infty}\left\|{ }_{a} D^{\varepsilon, k} T^{m} x_{a}-{ }_{a} D^{\varepsilon, k} x\right\|=0$. That is ${ }_{a} D^{\varepsilon, k} x \in C_{\gamma, k}[a, b]$ and $x \in C_{\gamma, k}^{\varepsilon, 0}[a, b]$.

Consider the initial value problem

$$
{ }_{a}^{C} D^{\varepsilon, k} x(t)=\lambda x(t)+f(t), t>a, x(a)=x_{a},
$$

where $\varepsilon \in(0,1), \lambda \in \mathbb{R}, f$ real-valued function and $k$ is an increasing and positive monotone function on $(a, b]$ which has a continuous derivative $k^{\prime}$ on $(a, b)$. Throughout this article $k$ will be assumed to have the previously mentioned properties.

Theorem 2. The solution of the Caputo initial value problem (28) is given by

$$
\begin{gathered}
x(t)=E_{\varepsilon}\left(\lambda(k(t)-k(a))^{\varepsilon}\right) x_{0}+ \\
\int_{a}^{t}(k(t)-k(s))^{\varepsilon-1} E_{\varepsilon, \varepsilon}\left(\lambda(k(t)-k(s))^{\varepsilon}\right) f(s) k^{\prime}(s) d s,
\end{gathered}
$$

where $E_{\varepsilon}($.$) and E_{\mathcal{\varepsilon}, \varepsilon}($.$) are the Mittag-Leffler functions of 1$ parameter and 2 parameters, respectively [3].

Proof. Consider the successive approximation

$$
4 x_{m}(t)=x_{a}+\lambda_{a} I^{\varepsilon, k} x_{m-1}(t)+{ }_{a} I^{\varepsilon, k} f(t), \quad m=1,2, \ldots, x_{0}(t)=x_{0} .
$$

Then, by Lemma 1 , by writing $x_{a}=x_{a}(k(t)-k(a))^{1-1}$ we have

$$
x_{1}(t)=x_{a}+\lambda \frac{\Gamma(1)}{\Gamma(\varepsilon+1)}(k(t)-k(a))^{\varepsilon} x_{a}+{ }_{a}{ }^{\varepsilon, k} f(t) .
$$


Proceeding inductively and by making use of Lemma 1, we have

$$
x_{m}(t)=x_{a} \sum_{i=0}^{m} \frac{\lambda^{i}(k(t)-k(a))^{i \varepsilon}}{\Gamma(k \varepsilon+1)}+\sum_{i=1}^{m} \lambda^{i-1}{ }_{a} I^{i \varepsilon, k} f(t) .
$$

Then, we reach our claim by expanding $t_{0} I^{k \varepsilon, k}$ in the second summation, shifting the index $k$, interchanging the order of the integral and summation, and letting $m \rightarrow \infty$.

Remark 6. The solution (29) coincides with the solution given in Theorem 5.2. in [17].

\section{Mittag-Leffler Bounds for Fractional Non-Autonomous Systems and $k$-Mittag-Leffler Stability}

Consider the system

$$
{ }_{a}^{C} D^{\varepsilon, k} x(t)=f(t, x), t>a, x(a)=x_{a},
$$

where $\varepsilon \in(0,1)$," and $f:[a, \infty) \times G \rightarrow \mathbb{R}^{n}$ is piecewise continuous in $t$ and locally Lipchitz in $x$ on $[a, \infty) \times G$ with Lipchitz constant $A>0$ and $G \subset \mathbb{R}^{n}$ is a domain that contains the origin $x=0$. Then we have

Definition 4. The constant $x_{0}$ is an equilibrium point of the system (33), if $f\left(t, x_{0}\right)=0$ and when ${ }_{a}^{C} D^{\varepsilon, k}$ is replaced by ${ }_{a} D^{\varepsilon, k}$ if ${ }_{a} D^{\varepsilon, k} x_{0}=f\left(t, x_{0}\right)$.

Definition 5. ( $k$-Mittag-Leffler stability) The solution $x(t)$ of the system (33) is called $k$-Mittag-Leffler stable if

$$
\|x(t)\| \leq\left(h\left(x_{0}\right) E_{\varepsilon}\left(\lambda(k(t)-k(a))^{\varepsilon}\right)\right)^{c},
$$

where $\lambda>0, c>0, h(0)=0, h(x) \geq 0$ and $h(x)$ is locally Lipchitz on on a ball B of $\mathbb{R}^{n}$ with Lipchitz constant $h_{0}$.

Lemma 4. For the continuous function $f(t, x)$ in (33), for any $\varepsilon>0$ and a certain norm of $\mathbb{R}^{n}$ we have

$$
\left\|I^{\varepsilon, k} f(t, x(t))\right\| \leq{ }_{a} I^{\varepsilon, k}\|f(t, x(t))\| .
$$

Proof. The proof is direct and similar to the proof of Lemma 2 in [27].

Theorem 3. The solution of system (33) satisfies

$$
\left.\|x(t)\| \leq\left\|x_{a}\right\| E_{\varepsilon}(A(k(t)-k(a)))^{\varepsilon}\right) .
$$

Proof. By applying the fractional integral ${ }_{a} I^{\varepsilon, k}$ to both sides of (33), and making use of Lemma 3 with $n=1$ we have

$$
x(t)-x(a)={ }_{a} I^{\varepsilon, k} f(t, x(t)) .
$$

If we apply the norm $\|$.$\| to (37), use Lemma 4$ and use the Lipchitz assumption we reach at

$$
|\|x(t)\|-\|x(a)\|| \leq\|x(t)-x(a)\|=\left\|_{a} I^{\varepsilon, k} f(t, x(t))\right\| \leq A_{a} I^{\varepsilon, k}\|x(t)\| .
$$

Find a nonnegative function $L(t)$ such that

$$
\|x(t)\|-\|x(a)\|=A_{a} I^{\varepsilon, k}\|x(t)\|-L(t) .
$$

By applying the following successive iteration in the frame of an existence uniqueness theorem similar to Theorem 1 


$$
y_{m}(t)=y_{0}+A_{a} I^{\varepsilon, k} y_{m-1}(t)-L(t), y_{0}(t)=\left\|x_{a}\right\|, y(t)=\|x(t)\|,
$$

and proceeding similar to what we have applied in Theorem 2, we conclude the solution representation

$$
\begin{aligned}
\|x(t)\| & =\left\|x_{a}\right\| E_{\varepsilon}\left(A(k(t)-k(a))^{\varepsilon}\right)-\int_{a}^{t} \sum_{i=0}^{\infty} \frac{1}{\Gamma(\varepsilon i)} A^{k}(k(t)-k(s))^{i \varepsilon-1} L(s) k^{\prime}(s) d s \\
& =\left\|x_{a}\right\| E_{\varepsilon}\left(A(k(t)-k(a))^{\varepsilon}\right)-\int_{a}^{t} T_{k} E_{\varepsilon}\left(A(k(t)-k(s))^{\varepsilon}\right) L(s) k^{\prime}(s) d s,
\end{aligned}
$$

where the operator $T_{k}=\frac{1}{k^{\prime}(t)} \frac{d}{d t}$. Since the second term in (41) is nonnegative, we conclude that

$$
\left.\|x(t)\| \leq\left\|x_{a}\right\| E_{\varepsilon}(A(k(t)-k(a)))^{\varepsilon}\right) .
$$

This completes the proof.

Remark 7. In Theorem 3, if $\varepsilon=1$, it follows that

$$
\|x(t)\| \leq\left\|x_{a}\right\| e^{A(k(t)-k(a))} .
$$

Now, we extend the Lyapunov direct method to the case of $k$-fractional-order systems.

Theorem 4. Let $x_{0}$ be an equilibrium point for the system (33) and $\mathbb{D} \subset \mathbb{R}^{n}$ be a domain containing $x_{0}$. Let $V(t, x(t)):[a, \infty) \times \mathbb{D} \rightarrow \mathbb{R}$ be a continuously differentiable function and locally Lipschitz with respect to $x$ such that

$$
\begin{gathered}
\beta_{1}\|x(t)\|^{\frac{1}{c}} \leq V(t, x(t)) \leq \beta_{2}\|x(t)\|^{d} \\
{ }_{a}^{C} D^{\varepsilon, k} V(t, x(t)) \leq-\beta_{3}\|x(t)\|^{d},
\end{gathered}
$$

$x \in \mathbb{D}, \varepsilon \in(0,1), \beta_{1} ; \beta_{2}, \beta_{3}, c$ and $d$ are arbitrary positive constants. Then, $x_{0}$ is $k$-Mittag-Leffler stable. If the assumptions hold globally on $\mathbb{R}^{n}$, then $x_{0}$ is globally $k$-Mittag-Leffler stable.

Proof. From (42) and (43), we obtain that

$$
{ }_{a}^{C} D^{\varepsilon, k} V(t, x(t)) \leq-\frac{\beta_{3}}{\beta_{2}} V(t, x(t))
$$

It follows that there exists a nonnegative function $L(t)$ such that

$$
{ }_{a}^{C} D^{\varepsilon, k} V(t, x(t))=-\frac{\beta_{3}}{\beta_{2}} V(t, x(t))-L(t) .
$$

Now, applying Theorem 1 or Theorem 5.2. in [17] (which was proved by the modified Laplace transforms), we get

$$
\begin{aligned}
V(t, x(t)) & =V\left(a, x_{a}\right) E_{\varepsilon}\left(-\frac{\beta_{3}}{\beta_{2}}(k(t)-k(a))^{\varepsilon}\right) \\
& -\int_{a}^{t}(k(t)-k(s))^{\varepsilon-1} E_{\varepsilon, \varepsilon}\left(-\frac{\beta_{3}}{\beta_{2}}(k(t)-k(s))^{\varepsilon}\right) L(s) k^{\prime}(s) d s
\end{aligned}
$$

Thus, we have

$$
V(t, x(t)) \leq V\left(a, x_{a}\right) E_{\mathcal{\varepsilon}}\left(-\frac{\beta_{3}}{\beta_{2}}(k(t)-k(a))^{\varepsilon}\right)
$$


Utilizing condition (42), we reach

$$
\beta_{1}\|x(t)\|^{\frac{1}{c}} \leq V\left(a, x_{a}\right) E_{\varepsilon}\left(-\frac{\beta_{3}}{\beta_{2}}(k(t)-k(a))^{\varepsilon}\right) .
$$

Therefore,

$$
\|x(t)\| \leq\left[\frac{V\left(a, x_{a}\right)}{\beta_{1}} E_{\varepsilon}\left(-\frac{\beta_{3}}{\beta_{2}}(k(t)-k(a))^{\varepsilon}\right)\right]^{c} .
$$

Hence, the system is $k$-Mittag-Leffler stable.

Using Remark 1 in [17] with $n=1$ or $\varepsilon \in(0,1)$ we have the following identity

$$
{ }_{a}^{C} D^{\varepsilon, k} x(t)={ }_{a} D^{\varepsilon, k} x(t)-\frac{x(a)}{\Gamma(1-\varepsilon)}(k(t)-k(a))^{-\varepsilon} .
$$

As a result of (44), For $\varepsilon \in(0,1)$ and $x(a) \geq 0$ we have ${ }_{a}^{C} D^{\varepsilon, k} x(t) \leq{ }_{a} D^{\varepsilon, k} x(t)$ and hence we can state the following stability theorem for the Riemann-Liouville generalized type fractional derivatives.

Theorem 5. Under the assumptions of Theorem 4, if we replace ${ }_{a}^{C} D^{\varepsilon, k} x(t)$ by ${ }_{a} D^{\varepsilon, k} x(t)$, we have that $x_{0}$ is globally k-Mittag-Leffler stable.

Remark 8. If in the above, we take $k(t)=t$, we recover the Mittag-Leffler stability for the systems of Caputo and Riemann-Liouville fractional derivatives. If we take $k(t)=\ln t$ then the sufficient conditions for the Mittag-Leffler stability of Caputo-Hadamard and Hadamard fractional systems are determined by Theorems 4 and 6 , respectively.

\section{The Lyapunov Direct Method in the Term of Comparison Functions}

In this section, we analyze the $k$-Mittag-Leffler stability using the Lyapunov direct method. The Lyapunov direct method presented in this section is done in the term of the comparison functions. The comparison functions play an essential role in the stability analysis. Before starting this section, we introduce some definitions related to the comparison functions $[37,38]$.

A continuous function $\alpha: \mathbb{R}_{\geq 0} \rightarrow \mathbb{R}_{\geq 0}$ is said to be positive definite when it satisfies the conditions $\alpha(0)=0$, and $\alpha(s)>0$ for all $s>0$. A class $\mathcal{K}$ denotes a set of all increasing functions which are positive definite functions.

The class $\mathcal{K}_{\infty}$ denotes a set of all unbounded $k$ functions. The continuous function $\beta: \mathbb{R}_{\geq 0} \times$ $\mathbb{R}_{\geq 0} \rightarrow \mathbb{R}_{\geq 0}$ is said to be class $\mathcal{K} \mathcal{L}$ if the function $\beta(., t) \in \mathcal{K}$ for any $t \geq 0$, is non-increasing and tends to zero as its arguments tend to infinity. For the characterization of the global $k$-Mittag-Leffler stability using the Lyapunov direct method, we introduce the following theorems which generalizes Theorem 4.

Theorem 6. Suppose there exists a positive continuous function $V: \mathbb{R}^{+} \times \mathbb{R}^{n} \longrightarrow \mathbb{R}$, class $\mathcal{K}_{\infty}$ functions $\alpha_{1}, \alpha_{2}, \alpha$ and a class $\mathcal{K} \mathcal{L}$ function $\beta$ satisfying the following assumptions

$$
\begin{gathered}
\alpha_{1}(\|x\|) \leq V(t, x) \leq \alpha_{2}(\|x\|) . \\
{ }_{a}^{C} D^{\varepsilon, k} V(t, x) \leq-\alpha(\|x\|) .
\end{gathered}
$$

If in addition the following assumption holds

$$
\alpha_{1}^{-1}\left(2 \beta\left(\left\|x_{a}\right\|,(k(t)-k(a))^{\varepsilon}\right)\right) \leq\left\|x_{a}\right\| E_{\varepsilon}\left(-c(k(t)-k(a))^{\varepsilon}\right)
$$

where $c$ is a positive constant. Then, the trivial solution of the $k$-fractional differential equation (33) is globally $k$-Mittag-Leffler stable. 
When the condition (45) holds the function $V$ is said to be radially unbounded. In other words, the function $V$ is said to be a Lyapunov candidate function when the assumption (45) holds. The assumption (47) represents the Mittag-Leffler estimate of the class $\mathcal{K} \mathcal{L}$ function and is essential to study the $k$-Mittag-Leffler stability.

Proof. The first assumption (45) implies the function $V$ is a Lyapunov candidate function and combining it with the second assumption (46), we have the following relationship

$$
{ }_{a}^{C} D^{\varepsilon, k} V(t, x) \leq-\alpha\left(\alpha_{2}^{-1}(V(t, x))\right) \text {. }
$$

From [39], there exists a class $\mathcal{K} \mathcal{L}$ function $\beta$ such that the following relationship is held

$$
\alpha_{1}(\|x\|) \leq V(t, x) \leq \beta\left(\left\|x_{a}\right\|,(k(t)-k(a))^{\varepsilon}\right)
$$

From which the following relationship follows

$$
\|x\| \leq \alpha_{1}^{-1}\left(2 \beta\left(\left\|x_{a}\right\|,(k(t)-k(a))^{\varepsilon}\right)\right) .
$$

Using the $k$-Mittag-Leffler estimate (47), we have the following identity

$$
\|x\| \leq \alpha_{1}^{-1}\left(2 \beta\left(\left\|x_{a}\right\|,(k(t)-k(a))^{\varepsilon}\right)\right) \leq\left\|x_{a}\right\| E_{\varepsilon}\left(-k(k(t)-k(a))^{\varepsilon}\right) .
$$

From which it follows the global $k$-Mittag-Leffler stability of the trivial solution of the fractional differential equation (33).

When the condition (45) holds the function $V$ is said to be radially unbounded. In other words, the function $V$ is said to be a Lyapunov candidate function when the assumption (45) holds. The assumption (47) represents the Mittag-Leffler estimate of the class $\mathcal{K} \mathcal{L}$ function and is essential to study the $k$-Mittag-Leffler stability.

Remark 9. The use of the Lyapunov direct method in the context of fractional order derivative is not trivial due to the fact that the determination of the generalized fractional derivative in the Caputo sense of a given function is not always possible to obtain. In many cases, we suppose the Lyapunov function for the fractional differential equation is quadratic. For these reasons, we propose the following assumption which is already proved in the literature in [40],

$$
{ }_{a}^{C} D^{\varepsilon, k} x^{T} P x \leq 2 x^{T} P{ }_{a}^{C} D^{\varepsilon, k} x,
$$

where $P$ represents a positive definite matrix. The dimension of the matrix $P$ depends on the dimension of the fractional differential equation (33).

\section{Examples}

In this section, we provided some examples to illustrate Theorems 4 and 6.

Firstly, we illustrate Theorem 4 by the following example. Let the k-fractional differential equation described by the generalized fractional derivative in the Caputo sense given by

$$
\left\{\begin{array}{l}
{ }_{a}^{C} D^{\varepsilon, k} x_{1}=-x_{1}+x_{2} \\
{ }_{a}^{C} D^{\varepsilon, k} x_{2}=-x_{2}
\end{array}\right.
$$

where $x^{T}=\left(x_{1}, x_{2}\right) \in \mathbb{R}^{2}$. Let the quadratic Lyapunov candidate function defined by $V(t, x)=$ $\frac{1}{2}\left(x_{1}^{2}+x_{2}^{2}\right)$. Combining Equation (52) and the generalized fractional derivative in Caputo sense of the Lyapunov function along the trajectories, we obtain the following relationships 


$$
\begin{aligned}
{ }_{a}^{C} D^{\varepsilon, k} V(t, x) \leq x^{T}{ }_{a}^{C} D^{\varepsilon, k} x & =-x_{1}^{2}+x_{1} x_{2}-x_{2}^{2} \\
& =-x_{1}^{2}+\frac{1}{2} x_{1}^{2}+\frac{1}{2} x_{2}^{2}-x_{2}^{2} \\
& =-\frac{1}{2} x_{1}^{2}-\frac{1}{2} x_{2}^{2} \\
& =-V(t, x) .
\end{aligned}
$$

It follows from Theorem 4, the trivial solution of the fractional differential Equation (53) is global $k$-Mittag-Leffler stable.

Secondly, we illustrate Theorem 6 by the following example. Let the fractional differential equation described by the generalized fractional derivative in the Caputo sense be given by

$$
\left\{\begin{array}{l}
{ }_{a}^{C} D^{\varepsilon, k} x_{1}=-x_{1}+x_{2}^{2} \\
{ }_{a}^{C} D^{\varepsilon, k} x_{2}=-x_{1} x_{2}-x_{2}
\end{array}\right.
$$

where $x^{T}=\left(x_{1}, x_{2}\right) \in \mathbb{R}^{2}$. Let the quadratic Lyapunov candidate function defined by $V(t, x)=$ $\frac{1}{2}\left(x_{1}^{2}+x_{2}^{2}\right)=\frac{1}{2}\|x\|^{2}$. Combining Equation (52) and the generalized fractional derivative in the Caputo sense of the Lyapunov function along the trajectories, we obtain the following relationships:

$$
\begin{aligned}
{ }_{a}^{C} D^{\varepsilon, k} V(t, x) \leq x^{T}{ }_{a}^{C} D^{\varepsilon, k} x & =-x_{1}^{2}+x_{1} x_{2}^{2}-x_{1} x_{2}^{2}-x_{2}^{2} \\
& =-x_{1}^{2}-x_{2}^{2}=-2 V(t, x) \\
& =-2 V(t, x) .
\end{aligned}
$$

Recalling Theorem 6 and the $\varepsilon$-integration of Equation (55), we obtain the following inequality

$$
\|x\| \leq\left\|V\left(x_{a}\right)\right\| E_{\varepsilon}\left(-2(k(t)-k(a))^{\varepsilon}\right) .
$$

Thus, the trivial solution of the $k$-fractional differential equation (54) is global $k$-Mittag-Leffler stable.

\section{Conclusions}

The fractional derivatives, in either Riemann-Liouville or Caputo settings, of function with respect to function, unify and extend Riemman-Liouville, Caputo, Hadamard, Caputo-Hadamard and other fractional derivatives. In this article, we proved the existence and uniqueness theorem for the generalized fractional derivative systems in newly defined function spaces. Then, we applied the successive approximation to obtain the solution representation for nonhomogeneous fractional differential systems with a constant coefficient. We employed these results to obtain Mittag-Leffler bounds for the solutions under Lipschitzity. The proved tools were used to prove $k$-Mittag-Leffler stability theorems of the Caputo and Riemann-Liouville types using a Lyapunov direct method. Alternatively, it is worth mentioning that the Laplace transforms introduced recently in [17] can be used to proceed in the proof of the main results instead of applying an operator method through successive approximations. Finally, we solved and discussed some examples. We should note that in the classical stability analysis, studying the global $k$-Mittag-Leffler stability using the analytical solution is not trivial and, in fact, almost impossible. An alternative issue is in using the Lyapunov direct method. The inconvenience of this proposed method is in finding the exact Lyapunov function for the given model. Another inconvenience of this method is in the calculation of the fractional derivative. To avoid these difficulties we propose using (52) which is fundamental in our studies.

Author Contributions: Conceptualization, T.A. and F.J.; methodology, F.J. and N.S.; formal analysis, F.M.; investigation, F.M.; resources, F.M.; writing-original draft preparation, T.A.; writing-review and editing, F.J. and N.S.; visualization, T.A.; supervision, N.S. 
Funding: The first author would like to thank Prince Sultan University for funding this work through research group Nonlinear Analysis Methods in Applied Mathematics (NAMAM) group number RG-DES2017-01-17.

Acknowledgments: All authors would like to thank their institutions for the technical supports.

Conflicts of Interest: The authors declare no conflict of interest.

\section{References}

1. Podlubny, I. Fractional Differential Equations; Academic Press: San Diego, CA, USA, 1999.

2. Samko, S.G.; Kilbas A.A.; Marichev O.I. Fractional Integrals and Derivatives: Theory and Applications; Gordon and Breach: Yverdon, Switzerland, 1993.

3. Kilbas, A.A.; Srivastava, H.M.; Trujillo, J.J. Theory and Application of Fractional Differential Equations; North Holland Mathematics Studies 204; Elsevier: Amsterdam, The Netherlands, 2006.

4. Magin, R.L. Fractional Calculus in Bioengineering; Begell House Publishers: Redding, CT, USA, 2006.

5. Hilfer, R. Applications of Fractional Calculus in Physics; Word Scientific: Singapore, 2000.

6. Luchko, Y. Fractiona wave equations and damped waves. J. Math. Phys. 2013, 54, 031505. [CrossRef]

7. Suzuki, J.L.; Zayernouri, M.; Bittencourt, M.L.; Karniadakis, G.E. Fractional-order uniaxial visco-elasto-plastic models for structural analysis. Comput. Meth. Appl. Mech. Eng. 2016, 308, 443-467. [CrossRef]

8. Rakhshan, M.; Gupta, V.; Goodwine, B. On passivity of fractional order systems. SIAM J. Control Optim. 2019, 57, 1378-1389. [CrossRef]

9. Ardjouni, A.; Djoudi, A. Existence and uniqueness of solutions for nonlinear implicit Caputo-Hadamard fractional differential equations with nonlocal conditions. Adv. Theor. Nonlinear Anal. Appl. 2019, 3, 46-52. [CrossRef]

10. Katugampola, U.N. New approach to a generalized fractional integral. Appl. Math. Comput. 2011, 218, 860-865. [CrossRef]

11. Katugampola, U.N. A new approach to generalized fractional derivatives. Bull. Math. Anal. Appl. 2014, 6,1-15.

12. Kilbas, A.A. Hadamard type fractional calculus. J. Korean Math. Soc. 2001, 38, 1191-1204.

13. Jarad, F.; Abdeljawad, T.; Baleanu, D. Caputo-type modification of the Hadamard fractional derivatives. Adv. Differ. Equ. 2012, 2012, 142. [CrossRef]

14. Gambo, Y.Y.; Jarad, F.; Baleanu, D.; Abdeljawad, T. On Caputo modification of the Hadamard fractional derivatives. Adv. Differ. Equ. 2014, 2014, 10. [CrossRef]

15. Jarad, F.; Abdeljawad, T.; Baleanu, D. On the generalized fractional derivatives and their Caputo modification. J. Nonlinear Sci. Appl. 2017, 10, 2607-2619. [CrossRef]

16. Adjabi, Y.; Jarad, F.; Baleanu, D.; Abdeljawad, T. On Cauchy problems with Caputo Hadamard fractional derivatives. J. Comput. Anal. Appl. 2016, 21, 661-681.

17. Jarad, F.; Abdeljawad, T. Generalized fractional derivatives and Laplace transform. Discret. Contin. Dyn. Ser. S 2019, 709-722. [CrossRef]

18. Meerschaert, M.M.; Zhang, Y.; Baeumer, B. Tempered anomlous diffusion in hetrogeneous systems. Geophys. Res. Lett. 2008, 35, L17403. [CrossRef]

19. Atangana, A.; Baleanu, D. New fractional derivatives with non-local and non-singular kernel: Theory and application to heat transfer model. Therm. Sci. 2016, 20, 753-763. [CrossRef]

20. Caputo, M.; Fabrizio, M. A new definition of fractional derivative without singular kernel. Prog. Fract. Differ. Appl. 2015, 1, 73-85

21. Losada, J.; Nieto, J.J. Properties of a new fractional derivative without singular kernel. Prog. Fract. Differ. Appl. 2015, 1, 87-92.

22. Abdeljawad, T.; Baleanu, D. Integration by parts and its applications of a new nonlocal fractional derivative with Mittag-Leffler nonsingular kernel. J. Nonlinear Sci. Appl. 2017, 10, 1098-1107. [CrossRef]

23. Abdeljawad, T.; Baleanu, D. Monotonicity results for fractional difference operators with discrete exponential kernels. Adv. Differ. Equ. 2017, 2017, 78 [CrossRef]

24. Abdeljawad, T.; Baleanu, D. On fractional derivatives with exponential kernel and their discrete versions. Rep. Math. Phys. 2017, 80, 11-27. [CrossRef] 
25. Jaishankar, A.; McKinley, G.H. Power-Law rheology in the bulk and at the interface: quasi-properties and fractional constitutive equations. Proc. R. Soc. A 2013. [CrossRef]

26. Li, Y.; Chen, Y.Q.; Podlubny, I. Stability of fractional-order nonlinear fractional systems: Lyapunov direct method and generalized Mittag-Leffler stability. Comput. Math. Appl. 2010, 59, 1810-1821. [CrossRef]

27. Li, Y.; Chen, Y.Q.; Podlubny, I. Mittag-Leffler stability of fractional order nonlinear dynamic systems. Automatica 2009, 45, 1965-1969. [CrossRef]

28. Jarad, F.; Abdeljawad, T.; Baleanu, D.; Biçen, K. On the Stability of Some Discrete Fractional Nonautonomous Systems. Abstr. Appl. Anal. 2012. [CrossRef]

29. Jarad, F.; Abdeljawad, T.; Baleanu, D. Stability of $q$-fractional non-autonomous systems. Nonlinear Anal. Real World Appl. 2013, 14, 780-784. [CrossRef]

30. Jarad, F.; Abdeljawad, T.; Baleanu, D.; Gündogdu, E. On the Mittag-Leffler stability of $q$-fractional nonlinear dynamical systems. Proc. Rom. Acad. Ser. A 2011, 12, 309-314.

31. Baleanu, D.; Sadati, S.J.; Ghaderi, R.; Ranjbar, A.; Abdeljawad, T.; Jarad, F. Razumikhin stability theorem for fractional systems with delay. Abstr. Appl. Anal. 2010. [CrossRef]

32. Sadati, S.J.; Baleanu, D.; Ranjbar, A.; Ghaderi, R.; Abdeljawad, T. Mittag-Leffler stability theorem for fractional nonlinear systems with delay. Abstr. Appl. Anal. 2010. [CrossRef]

33. Baleanu, D.; Ranjbar, A.; Sadati, S.J.; Delvari, R.H.; Abdeljawad, T.; Gejji, V. Lyapunov-Krasovskii stability theorem for fractional systems with delay. Rom. J. Phys. 2011, 56, 636-643.

34. Zhang, X. Some results of linear fractional order time delay systems. Appl. Math. Comput. 2008, 197, 407-411. [CrossRef]

35. Deng, W.; Li, C.; Lü, J. Stability analysis of linear fractional differential system with multiple time delays. Nonlinear Dyn. 2007, 48, 409-416. [CrossRef]

36. Momani, S.; Hadid, S. Lyapunov Stability solutions of fractional integrodifferential equations. Int. J. Math. Math. Sci. 2004, 47, 2503-2507. [CrossRef]

37. Sene, N.; Srivastava, G. Generalized Mittag-Leffler Input Stability of the Fractional Differential Equations. Symmetry 2019, 11, 608. [CrossRef]

38. Sene, N. Stability analysis of the generalized fractional differential equations with and without exogenous inputs. J. Nonlinear Sci. Appl. 2019, 12, 562-572. [CrossRef]

39. Sene, N. Exponential form for Lyapunov function and stability analysis of the fractional differential equations. J. Math. Comput. Sci. 2018, 18, 388-397. [CrossRef]

40. Aguila-Camacho, N.; Duarte-Mermoud, M.A.; Gallegos, J.A. Lyapunov functions for fractional order systems. Commun. Nonlinear Sci. Numer. Simul. 2014, 19, 2951-2957. [CrossRef]

(C) 2019 by the authors. Licensee MDPI, Basel, Switzerland. This article is an open access article distributed under the terms and conditions of the Creative Commons Attribution (CC BY) license (http://creativecommons.org/licenses/by/4.0/). 